\title{
The Catalytic Activity of Zeolite in Rare Earth Form for Isomerization and Disproportionation of Xylene*
}

\author{
by Hiroshige Matsumoto** and Yoshiro Morita**
}

\begin{abstract}
Summary: The catalytic activities of synthetic zeolites containing various amounts of rare earth cations were investigated in the isomerization and disproportionation of o-xylene using a flow apparatus. The rates of both reactions increased significantly with the increase in content of the rare earth cation and were proportional to the amount of water adsorbed on the surface.

The activation energy of isomerization was independent of rare earth content and of water content, while that of disproportionation increased with the decrease of rare earth and water contents.

From these observations, the active sites of these zeolite catalysts were proposed to be protons formed between the rare earth cations and the water molecules adsorbed on zeolite.
\end{abstract}

\section{Introduction}

In the study of a solid catalyst, one of the important obstructions is the heterogeneity of the active surface. However, zeolites, especially synthetic zeolites, have welldefined crystal surfaces which can be regarded as essentially homogeneous. For the catalytic sites of cationic zeolite in reactions of hydrocarbons, the "electrostatic field" theory was proposed by Rabo et al. ${ }^{1}$. The field originating from the zeolitic cation and the anionic framework was estimated to be strong enough to polarize hydrocarbon molecules which perform reaction intermediates. There is, however, no necessity to consider that the nature of active sites on crystalline alminosilicates should be basically different from that on amorphous materials, which show almost the same properties in catalysis. Furthermore, sodium zeolites, which seem to have only weak fields, show high catalytic activities upon the introduction of hydrogen chloride ${ }^{2)}$.

Since the presence of protonic sites has been confirmed on the zeolite in cationic forms ${ }^{3)}$, we consider the active sites to be the protonic sites formed between the zeolitic cation and the water molecule in a manner similar to that of the zeolitehydrogen chloride system. Namely, it is presumed that the cations do not polarize reactant moleculcs directly but instead polarize water molecules to produce active protons. In this work, the activities of rare earth zeolites for isomeriza-

* Recieved November 14, 1967.

** Department of Applied Chemistry, School of Science and Engineering, Waseda University (Nishiokubo, Shinjuku-ku, Tokyo, Japan). tion and disproportionation were investigated on the basis of this model of active sites.

\section{Experimental}

The original sodium form of zeolite used in this work was X-type Molecular Sieves (13X) from the Linde Company. The zcolites in rare earth form were obtained by the ion exchanges of $\mathrm{La}^{+++}$and $\mathrm{Ce}^{+++}$for $\mathrm{Na}^{+}$. The exchange levels of CeX, defined as the percent of the original $\mathrm{Na}^{+}$replaced, were determined to be 9,19 , 41, 59 and 94, and that of LaX was 95. The reaction of 0 -xylene was carried out using the same apparatus in the same manner as was reported previously). The amount of adsorbed water on the zeolite was measured by a thermobalance in the carrier gas (dried hydrogen) at high temperatures similar to that of the activity test.

\section{Results}

In the temperature range of $250 \sim 400^{\circ} \mathrm{C}$, the two types of reactions simultaneously proceed in the conversion of $o$-xylene catalyzed by zeolite. One of these is isomerization yielding $m$ - and $p$-xylenes (the migration of the methyl group along the aromatic ring), and the other is disproportionation to toluene and trimethylbenzenes (the intermolecular migration of the methyl group). The rates of these reactions can be treated independently by first order approximation as shown in the previous work. ${ }^{4)}$

According to the model for the active site on zeolite catalyst proposed in the zeolite-hydrogen chloride system, ${ }^{2)}$ the catalytic activity of zeolite in the absence of hydrogen chloride should be 


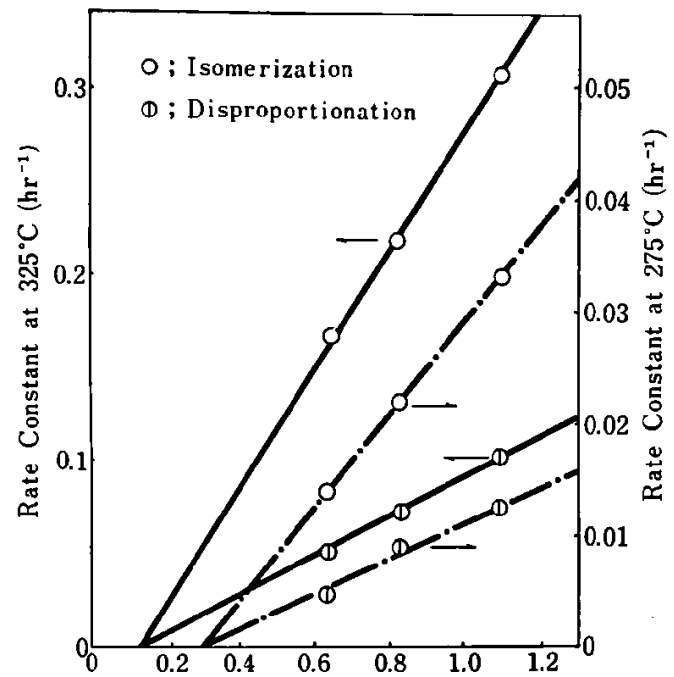

Amount of Adsorbed Water on $\mathrm{LaX}(\mathrm{mmol} / \mathrm{g}$ )

Fig. 1 Rate Constants of Isomerization and Disproportionation Versus Water Content on Zeolite

zeolitic cation. The results of thermogravimetric analysis showed that the LaX zeolite contained $9.8,7.4$ and $5.8 \mathrm{mg} / \mathrm{g}$ of water at 400,450 and $500^{\circ} \mathrm{C}$, respectively, in a strcam of dried hydrogen, while a great portion of the adsorbed water (probably, physically adsorbed water) was removed below $200^{\circ} \mathrm{C}$. The activity of $\mathrm{LaX}$ for the $o$-xylene reaction was then examined for its relationship to the water content which was controlled by pretreatment temperature. In Fig. 1, the rate constants of the isomerization and the disproportionation reactions are plotted as a function of water content of LaX. As is evident from Fig. 1, the rates of these reactions are proportional to the amount of water on the zeolite within a restricted region of adsorption. An extrapolation of the line of rate constantwater content, does not intercept the origin of

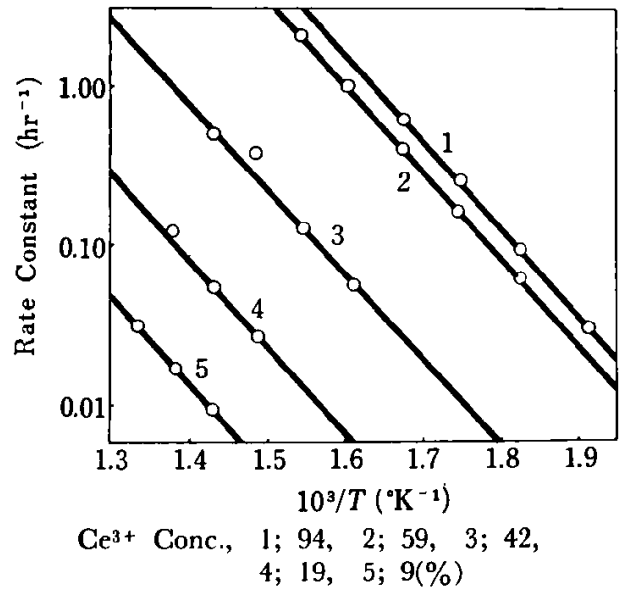

Fig. 2 Arrhenius Plots for Isomerization on CeX with Variation of Cerium Ion Concentration due to the interaction between water and the the figure. This discrepancy from the origin of the figure is apparentey due to a quantity of adsorbed water which is ineffective for the catalysis. From the intercepts of the horizontal axis of Fig. 1, it can be determined that the amounts of the ineffective water for the isomerization reaction agree well with those for the disproportionation reaction and arc cstimated to be 0.1 and $0.3 \mathrm{mmol} / \mathrm{g}$ at reaction temperatures of 325 and $275^{\circ} \mathrm{C}$, respectively.

At these reaction conditions, the zeolite in alkaline form was completely inactive for the reaction of 0 -xylene. Aftcr substituting the sodium ion with a polyvalent cation, such as a rare earth cation, zeolite showed a catalytic activity which increased with the increase of the exchange level. Thus, the amounts of active cation and water are considered to bc the most important factors in the catalytic activity of zeolites.

In the first place, the effect of these factors on the isomerization of $o$-xylene was investigated. A series of samples with increasing cerium ion concentration in sodium zcolite was examined. The results are shown in Fig. 2 with a plot of the logarithmic rate constant versus the reciprocal reaction temperature. The data show linear relationships and the same activation energy. Fig. 3 shows similar data for the series of LaX containing $5.8,7.4$ and $9.8 \mathrm{mg} / \mathrm{g}$ of water. The activation energy of the isomerization reaction are all the same in this case.

The same examinations were subsequently carried out for the disproportionation of 0 -xylene. Though the Arrhenius plots of the disproportionation reaction show good linearities (Fig. 4 and Fig. 5), their slopes are different from each other with respect to the concentration of cerium

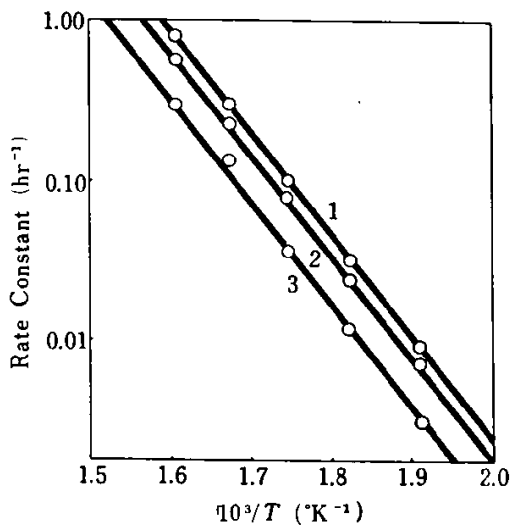

Water Content, 1; 9.8, 2; 7.4, 3; $5.8(\mathrm{mg} / \mathrm{g})$

Fig. 3 Arrhenius Plots for Isomerization on $\mathrm{LaX}$ with Variation of Water Content 


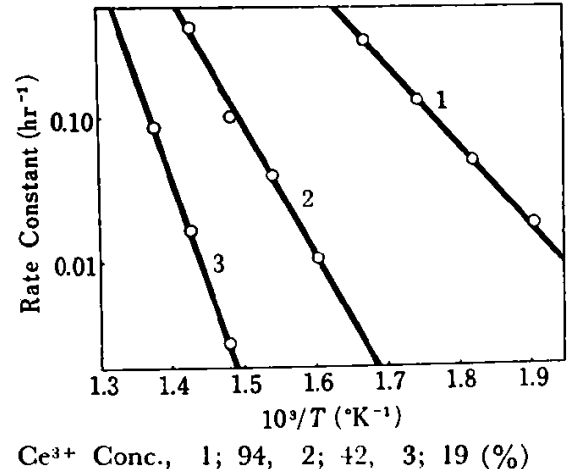

Fig. 4 Arrhenius Plots for Disproportionation on CeX with Variation of Ccrium Ion Concen. tration

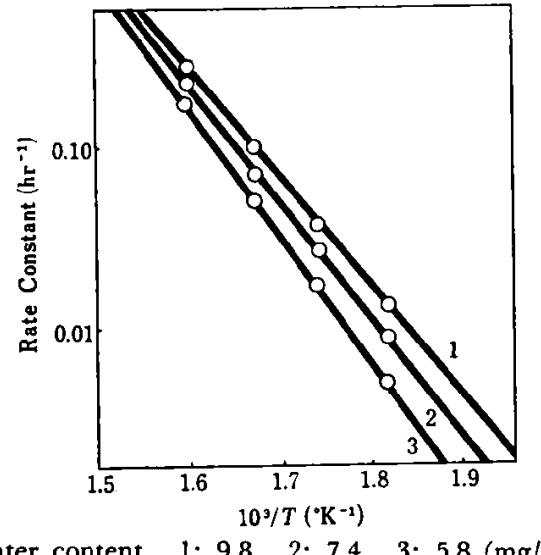

Water content, $1 ; 9.8,2 ; 7.4,3 ; 5.8(\mathrm{mg} / \mathrm{g})$

Fig. 5 Arrhenius Plots for Disproportionation on LaX with Variation of Water Content

ion and the water content of zeolite used. With the increase in content of rare earth cation and adsorbed water, the activation energy of the disproportionation reaction clearly decreased.

\section{Discussion}

Both the isomerization and disproportionation of 0 -xylene are apparently promoted by protonic acid, since the aprotonic acids such as alumina are completely inactive in contrast with protonic silica alumina which has a high activity under similar conditions. The zeolites adsorb a large amount of water and only part of that water can be expelled by conventional pretreatment with carrier gas. The remaining water is considered to play an important role in the catalysis. For the catalytic activity of zeolite, the action of this water has a strong resemblance to hydrogen chloride. That is, the activity of rare earth zeolite is reversibly enhanced by steaming the system $^{5)}$ and is proportional to the water content of the zeolite (Fig. 1). Therefore, the active site of the zeolite-water system is considered to be produced by the reversible interaction between the water moleculc and the zeolitic cation in much the same manner as the case of the zeolitehydrogen chloride system. In the acid-catalyzed reactions of aromatic hydrocarbons, however, the zeolites in alkaline forms are virtually inactive, even in the presence of considerable amounts of water, while hydrogen chloride strongly activates these zcolites. From these points of view, the catalytic surface of rare earth-sodium zeolite is regarded as the following simple model.

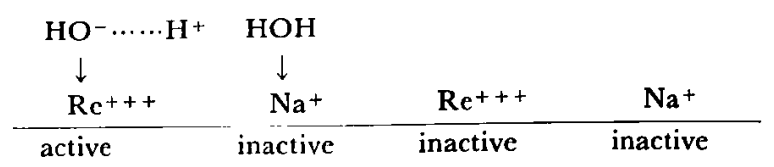

In the scheme of active protonic site formation, where the water molecule (Lewis base) interacts with the zeolitic cation (Lewis acid), the acid strength of the sodium ion will not be strong enough to polarize the water molecule. This model is not so contradictory to the results of IR measurements reported by $\mathrm{Ward}^{3)}$ and Shiba ${ }^{6}$. With the increase of evacuating temperature, the absorption band corresponding to the piridinium ion (near $1,540 \mathrm{~cm}^{-1}$ ) decreased in intensity and that corresponding to the coordinately bonded piridine (near $1,450 \mathrm{~cm}^{-1}$ ) increased in the spectra of piridine on rare earth or alkaline earth zeolite. Shiba also observed the absence of protonic acid on sodium zeolite even with the addition of water.

The activation energies of the isomerization and disproportionation reactions are shown in Fig. 6 and Fig. 7, in terms of their dependence on the contents of rare earth cation and water of zeolite, respectively. Recognizing the above model for the active site, the activation energy of isomerization was independent of the concentration of active sites on the surface. Therefore,

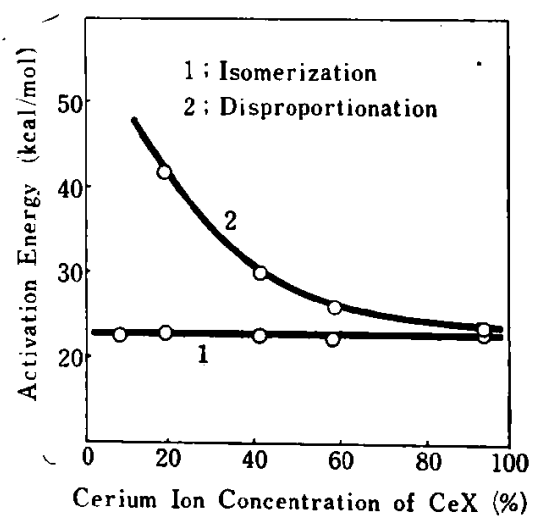

1 ; isomerization 2 ; disproportionation

Fig. 6 Activation Energies of Isomerization and Disproportionation Depending on Rare Earth Ion Concentration of Zeolite. 


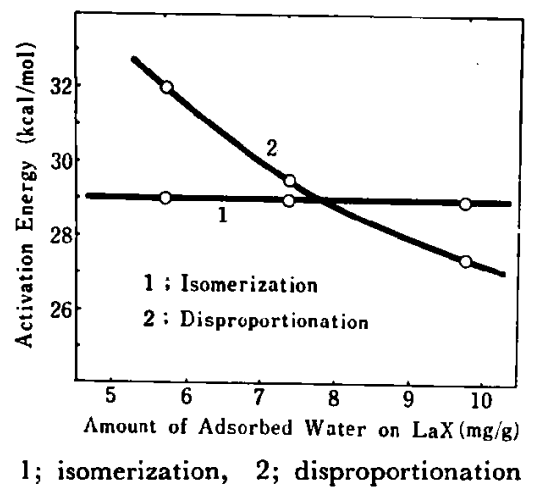

Fig. 7 Activation Energies of Isomerization and Disproportion Depending on Water Content of Zeolite

the nature of the active site does not appear to be affected by a change in the active site concentration (homogeneous surface for the isomerization).

On the other hand, the activation energy of disproportionation increased with the decrease of active site concentration. This doesn't necessarily imply heterogeneity of the active surface. The sites which are active for isomerization may be also available for disproportionation, since the amounts of water ineffective for the former were equal to that for the latter (from the intercepts of the horizontal axis in Fig. 1). With an increase in the concentration of active sites, the average distance between the sites should increase. Con- sequently, in the disproportionation reaction, higher energy will be required for the interaction between two molecules activated on these sites (Hinshelwood mechanism). On the other hand, one reaction occur only on one site in isomerization and is regarded as one molecular reaction. Therefore, the activation energy of isomerization will be independent of the distance between the sites.

These considerations are consistent with Echigoya's work ${ }^{7)}$ which reports that the disprcportionation of toluene on amorphous silicaalumina was able to be treated with the Hinshelwood kinetics.

\section{Reference}

1) Pickert, P. E., Rabo, J. A., Dempsey, E., Schomaker, V., Proc. Intern. Congr. Catalysis, 3rd, Amsterdam, 1964 2, 1264 (Wiley, New York, 1965).

2) Matsumoto, H., Yasui, K., Morita, Y., J. Catalysis, 12, 84 (1968).

3) Ward, J. W., ibid., 10, 34 (1968).

4) Matsumoto, H., Morita, Y., Bull. Japan Petrol. Inst., 10, 8 (1968).

5) Yamamoto, N., Fujii, K., Idemaru, Y., Furukawa, T., ibid., 8, 7 (1966).

6) Shiba, T.. Japan-USA Seminar on Catalytic Science, J-6 1968, Tokyo.

7) Echigaya, E., Masai, M., Saito, S., Murata, K., Nakamura, T., Morikawa, K., Kagaku Kögaku 31, 386 (1967). 